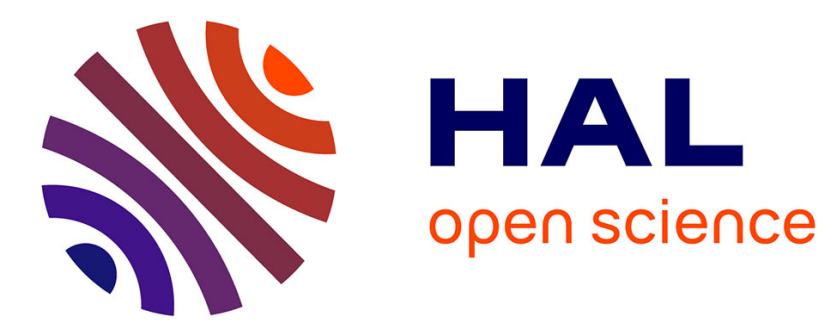

\title{
Experimental study of hydraulic transport of large particles in horizontal pipes
}

Florent Ravelet, Farid Bakir, Sofiane Khelladi, Robert Rey

\section{To cite this version:}

Florent Ravelet, Farid Bakir, Sofiane Khelladi, Robert Rey. Experimental study of hydraulic transport of large particles in horizontal pipes. Experimental Thermal and Fluid Science, 2013, 45, pp.187. 10.1016/j.expthermflusci.2012.11.003 . hal-00631562v3

HAL Id: hal-00631562

https://hal.science/hal-00631562v3

Submitted on 20 Nov 2012

HAL is a multi-disciplinary open access archive for the deposit and dissemination of scientific research documents, whether they are published or not. The documents may come from teaching and research institutions in France or abroad, or from public or private research centers.
L'archive ouverte pluridisciplinaire HAL, est destinée au dépôt et à la diffusion de documents scientifiques de niveau recherche, publiés ou non, émanant des établissements d'enseignement et de recherche français ou étrangers, des laboratoires publics ou privés. 


\title{
Experimental study of hydraulic transport of large particles in horizontal pipes
}

\author{
F. Ravelet ${ }^{\mathrm{a}, *}$, F. Bakir ${ }^{\mathrm{a}}$, S. Khelladi ${ }^{\mathrm{a}}$, R. Rey ${ }^{\mathrm{a}}$ \\ ${ }^{a}$ Arts et Metiers ParisTech, DynFluid, 151 boulevard de l'Hôpital, 75013 Paris, France.
}

\begin{abstract}
This article presents an experimental study of the hydraulic transport of very large solid particles (above $5 \mathrm{~mm}$ ) in an horizontal pipe. Two specific masses are used for the solids. The solids are spheres that are large with respect to the diameter of the pipe (5,10 and 15\%) or real stones of arbitrary shapes but constant specific mass and a size distribution similar to the tested spherical beads. Finally, mixtures of size and / or specific mass are studied. The regimes are characterized with differential pressure measurements and visualizations. The results are compared to empirical models based on dimensionless numbers, together with 1D models that are based on mass and momentum balance. A model for the transport of large particles in vertical pipes is also proposed and tested on data available in the Literature, in order to compare the trends that are observed in the present experiments in a horizontal pipe to the trends predicted for a vertical pipe. The results show that the grain size and specific mass have a strong effect on the transition point between regimes with a stationary bed and dispersed flows. The pressure drops are moreover smaller for large particles in the horizontal part contrary to what occurs for vertical pipes, and to the predictions of the empirical correlations.
\end{abstract}

Keywords: Hydraulic transport, solid-liquid two-phase flow, bed friction, deep sea mining.

\section{Introduction}

The hydraulic transport of solid particles is a method widely used in chemical and mining industries. Many predictive models exist in the case of slurries transported under homogeneous regime, that is to say when the particle diameter is small compared to the flow length scales and the velocity of the carrier fluid is high compared to the settling velocity of a particle [1 4] . It is then possible to predict the pressure losses in horizontal or vertical pipes with sufficient accuracy. In recent years, the sharp increase in demand for raw materials makes it interesting exploitation of new resources, particularly the use of fields at the bottom of the ocean [ [5, 6 . In this case, the solids may be large with respect to the pipe diameter and the circuit would have complex shapes, including vertical parts, horizontal parts, and potentially bends and S-shapes in order to absorb the deformations caused by surface waves. For transport of large particles in vertical pipe, a predictive model based on the work of Newitt et al. 7] and Richardson et al. [8] is proposed and validated on a set of experimental data [9 11]. However, in horizontal, and a fortiori in geometries in S-shape, there are few models [1 4, 12 - 15] and the effects of specific mass and more specifically of very large particle size have not been systematically explored. One major difficulty in the case of transport of large particles and high specific mass comes from the various flow regimes that may be observed [1, 2, 4, 12, 13, 16, 17]: when the speed of transportation increases, several transitions arise from regimes with a layer of solids at the bottom of the pipe that is at rest or that flows backwards in inclined pipes 13, 16, 17] to regimes with a moving bed and eventually to heterogeneous and pseudo-homogeneous suspensions at high mixture velocities.

The knowledge of the velocity above which the bed starts to move forward is of great interest with respect to operation of a production line. Below this limit the system may indeed plug. In the present study, experiments are carried out in order to better understand the effects of solid size and specific mass on this velocity and on the pressure drop.

The experimental set-up is presented in $\S 2$ Some general considerations on the typical regimes and pressure drop curves that are specific to an horizontal pipe and an overview of few models are presented in $\S 3$. The experimental results are presented and discussed in $\S$ 4. The main results that concern mono-disperse calibrated solid spheres of two different sizes and two different specific masses flowing in the horizontal pipe are presented in $\S$ 4.1. Mixture of spheres and rough stones of arbitrary shapes are also tested in $\S 4.2$, in order to check to what extent the results obtained for spheres may represent an actual application. Several models are proposed and compared to the experiments in $\S 4.3$. Conclusions and perspectives are then given in $\S$.

\footnotetext{
*corresponding author

Email address: florent.ravelet@ensta.org (F. Ravelet)
} 

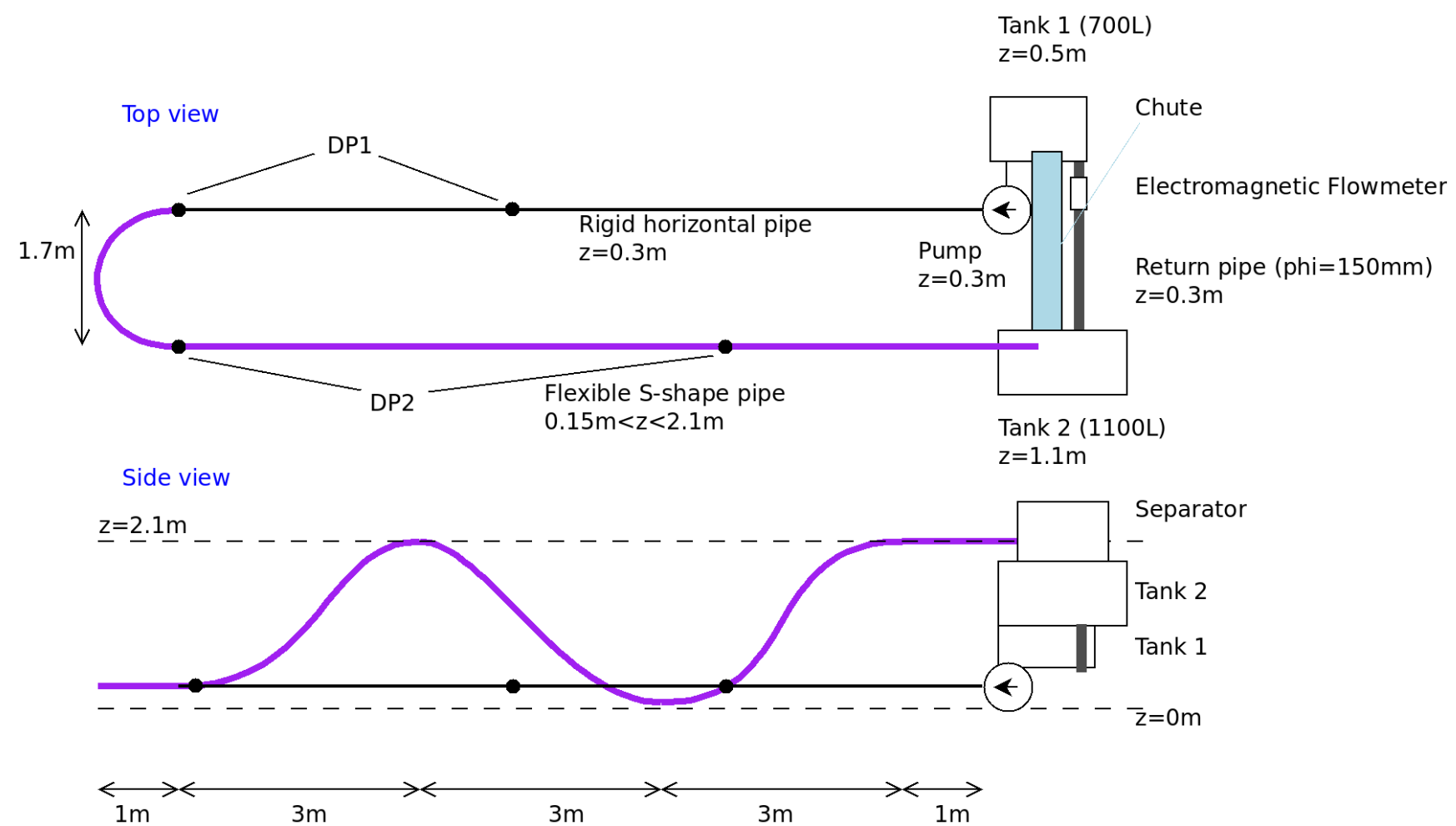

Figure 1: Sketch of the test loop. Top and side views.

\section{Experimental set-up}

\subsection{Test loop}

The test loop is shown schematically in Fig. 1. It consists of a first rigid, transparent horizontal pipe of internal diameter $D=100 \mathrm{~mm}$ and $10 \mathrm{~m}$ long in which flows the liquid/solid mixture. The return occurs in a clear flexible PVC hose reinforced with a steel coil, of internal diameter $D=100 \mathrm{~mm}$ and total length $20 \mathrm{~m}$. This return pipe first follows a $180^{\circ}$ horizontal curve of curvature diameter $1.7 \mathrm{~m}$, then a climb, a descent and an ascent in a vertical plane. The present study focuses on the horizontal pipe.

The mixture of solids and water that flows in the rigid horizontal pipe and the return flexible pipe is then passed through a separator consisting of a closed box fitted with a side hatch and whose bottom side consists of a grid of stainless steel. The water then falls into the tank 2 , and the solids flow in a chute that is inclined at $45^{\circ}$. This chute provides at its end an adjustable flap in order to control the solids flow rate. The end of the chute is above a grid container of known capacity that is immerged in tank 1 and connected with a grid pipe to the outlet of tank 1 . The separated water flows from tank 2 into tank 1 through a pipe with an electromagnetic flow-meter. The mixture is sucked by a vortex pump (Ensival Moret MT 100-100250) connected to the outlet of tank 1 and delivered in the circuit.

\subsection{Characteristics of the solids}

Solids with different sizes and specific masses are used. Their physical and geometrical characteristics are summarized in Tab. 1. The particles are relatively large, with sizes ranging from $5 \%$ to $18 \%$ of the pipe diameter. Calibrated beads of Glass (SiLi, SiLibeads type M, with a relative dispersion of sizes of 4\%) and of Alumina (Umicore, Alumina Degussit $92 \%$, with a relative dispersion of sizes of $10 \%$ ) are used. The real solids that are used from the perspective of an actual application have irregular shapes, as can be seen in the picture in Tab. 1. The specific mass of a sample of fifty solids have been measured with a densimeter. It is constant within $2700 \pm 10 \mathrm{~kg} \cdot \mathrm{m}^{-3}$.

\subsection{Control parameters and measured quantities}

The aim of the present work is to measure the pressure drops in different parts of the test loop as a function of concentration and velocity. Several choices can be made for the definition of these quantities. The natural control parameters, i.e. the parameters that are really adjusted with experimental means, are the volumetric flow rates of the liquid $\left(Q_{l}\right)$ and of the solids $\left(Q_{s}\right)$.

The following set of parameters is chosen for presenting the results. The first is the mixture velocity $V_{m i x}$ and the second is the transport or delivered concentration $C$ :

$$
\begin{gathered}
V_{\text {mix }}=\frac{Q_{l}+Q_{s}}{A} \\
C=\frac{Q_{s}}{Q_{s}+Q_{l}}
\end{gathered}
$$


with $A$ the cross-section area of the pipe. This mixture velocity is a volumetric average of the velocities of each phase. It is convenient for comparison with models.

Concerning the concentration, please note that on the one hand, the solids do not flow with the same velocity as water, because of their large size and of the large density ratio -in other words there is a non-negligible slip velocity. On the other hand, in horizontal parts, among the various regimes that may be observed there exist regimes with a stationary layer of solids at rest at the bottom of the pipe [4, 12, 13]. There could thus be a great difference between the transport or delivered concentration $C$ and the in-situ, local or volumetric concentration $\epsilon_{s}$ that is the ratio between the area occupied by the solids and the area of the pipe. Further, the volumetric concentration is a key parameter in the modelisation, as illustrated in $\oint 3.1$ and $\oint 3.3$. This concentration is however not a control parameter but results from the geometry, the physical properties of the phases and the boundary conditions.

The present experimental study is to perform measurements with varying $V_{m i x}$ in the range $0-5 \mathrm{~m} . \mathrm{s}^{-1}$, for constant delivered concentrations of $5,10,15$ et $20 \%$.

The water flow-rate is measured using an electromagnetic flowmeter (KROHNE Optiflux 2000) and adjusted by varying the rotation rate of the vortex pump. The solids flow-rate is set through a hatch and is measured by filling the buffer zone of known capacity located in the tank 1 . Finally, the pressure drop are measured using two differential pressure sensors (VEGADIF65): a first that is located at the end of the straight line, 60 diameters downward of the pump, and a second that is located at the hose in the S-shaped part (see the position of the pressure taps in Fig. 11). After a stationary state is reached, the data are recorded for $30 \mathrm{~s}$ at a sample rate of $130 \mathrm{~Hz}$. Measuring the rate of fluctuation of flow and pressure is used as a validation criterion of the measurements. The losses are expressed in terms of hydraulic gradients (meters of water column per meter of pipe) and with a dimensionless pressure drop coefficient:

$$
\begin{aligned}
& I(m / m)=\frac{\Delta P}{\rho_{l} g L} \\
& \lambda=\frac{\Delta P}{\frac{1}{2} \rho_{l} V_{m i x}^{2}} \frac{D}{L}
\end{aligned}
$$

with $\Delta P$ the measured static pressure drop, $\rho_{l}$ the specific mass of the carrying liquid (water) and $L$ the curvilinear distance between the pressure taps. The velocity scale that is used to present dimensionless results is the terminal velocity of the particle falling in the fluid at rest $V_{0}$. Finally, the relative specific mass of the solids with respect to water is $s=\frac{\rho_{s}}{\rho_{l}}$.

In order to compare the present data to existing theories and correlations [4, 15], the following quantity is also introduced:

$$
\Phi=\frac{I-I_{f}}{I_{f}}
$$


with $I_{f}$ the hydraulic gradient that would be observed for water flowing alone at the mixture velocity. It corresponds to the dimensionless excess of pressure drop caused by the presence of the particles. Similarly, two dimensionless groups are introduce for the mixture velocity:

$$
F_{D}=V_{m i x} / \sqrt{g D}
$$

a "Froude" number based on the mixture velocity and the pipe diameter and

$$
F_{d_{p}}=V_{0} / \sqrt{g d_{p}}
$$

a "Froude" number based on the terminal velocity and the size of the particle.

In the following, $I_{h}$ stands for the hydraulic gradient in the horizontal line, and $I_{s}$ stands for the hydraulic gradient in the S-shape pipe. The case of the S-shape part is not fully developed here and will be discussed in a forthcoming paper with new experimental data including a better discretisation of the different inclined parts and bends. The symbol $I_{v}$ is used for the hydraulic gradient that would be observed in a vertical pipe.

Optical measurements are also performed with a highspeed camera (Optronis CamRecord600). Typically 3200 images are recorded with a resolution of $1280 \times 1024$ pixels at a frame rate of $200 \mathrm{~Hz}$. The flow is illuminated backwards with a LED plate from Phlox. The visualization area is surrounded by a square plexiglas box filled with water in order to minimize optical distorsions.

\section{Observed regimes and overview of a few models}

A common feature of multiphase flows is the existence of very different flow patterns, which makes modeling much more complicated. In the case of the transport of large solid particles by a carrying liquid, several intermittent, stratified and dispersed regimes are observed in horizontal and inclined flows [1, 2, 4, 17]. For the specific case of vertical ascending flows, though plug or slug flows are observed in the pneumatic transport of small particles [18], to the best of our knowledge only dispersed regimes are observed for the hydraulic transport of large $\left(d_{p} \geqslant 1 \mathrm{~mm}\right)$ and massive $(s \geqslant 1.5)$ particles [2, 9 11]. The present section first presents a model for vertical ascending flows and then the typical regimes that are encountered in the horizontal experiment and the different possible models.

\subsection{Validation of a model for the hydraulic transport of large particles in ascending vertical pipelines}

The case of vertical flow is reasonably straightforward. The pressure force exerted on a column of fluid of height $z$ balances two forces: the hydrostatic weight of the mixture and the friction on pipe wall due to the fluid shear stress [1, 2]. In the following, the hydrostatic weight of the column of water is removed in order to present the hydraulic gradients that are due to the flow of a mixture in the pipe.
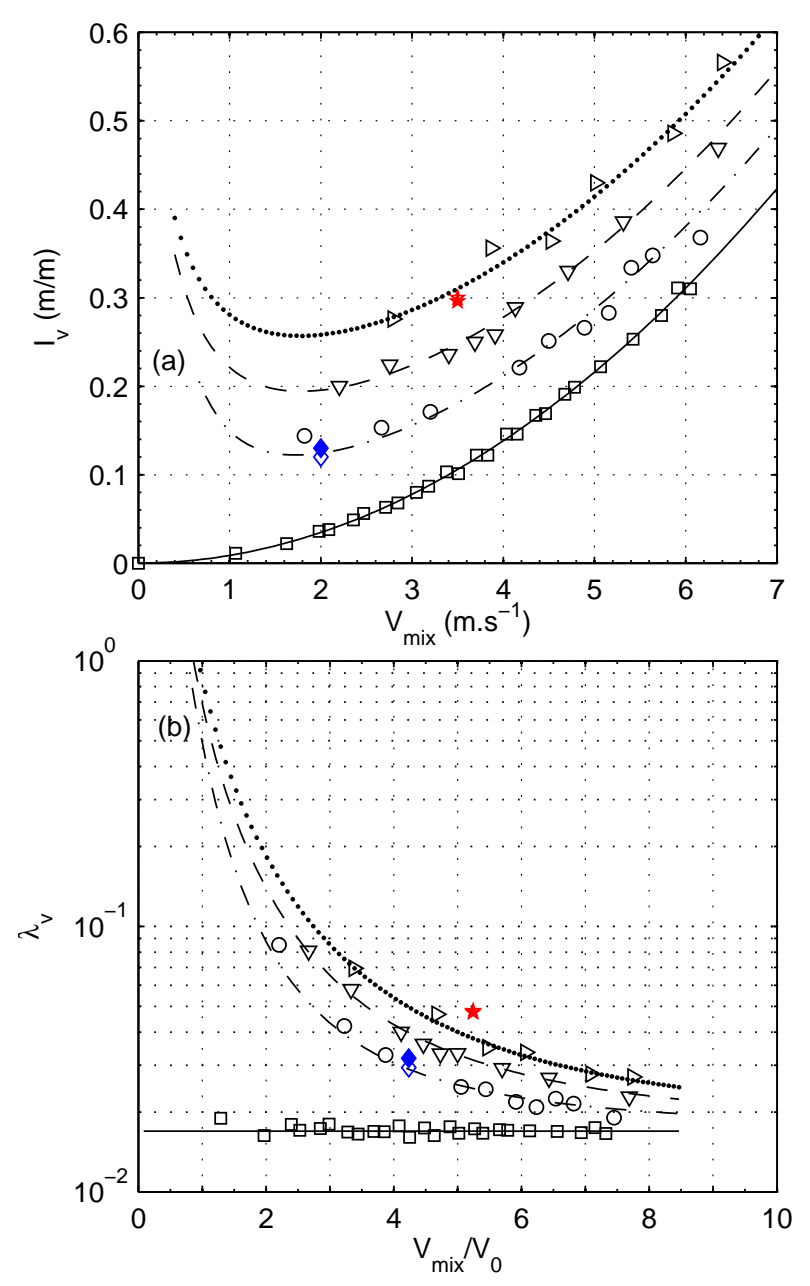

Figure 2: Validation of the vertical model on different data sets. Vertical hydraulic gradient $I_{v}$ vs. mixture velocity $V_{m i x}$. Black symbols: data of Yoon et al. [11] for spherical beads of diameter $d_{p}=20 \mathrm{~mm}$, $s=2.15$ flowing in a pipe of diameter $D=100 \mathrm{~mm}$, compared to the results of the model (black lines) for various concentrations ( $\square$ and filled line: $C=0 \%$, $\circ$ and dash-dotted line: $C=5 \%, \nabla$ and dashed line: $C=10 \%$ and $\triangleright$ and dotted line: $C=15 \%$ ). Red $\star$ : data (open symbol) of Xia et al. [9] for spherical beads of diameter $d_{p}=15 \mathrm{~mm}$, $s=2$ flowing in a pipe of diameter $D=100 \mathrm{~mm}$, at $C=15 \%$, compared to the result of the model (closed symbol). Blue $\diamond$ : data (open symbol) of Hong et al. [10] for spherical beads of diameter $d_{p}=5 \mathrm{~mm}, s=2.5$ flowing in a pipe of diameter $D=50 \mathrm{~mm}$, at $C=3.85 \%$, compared to the result of the model (closed symbol).

The vertical hydraulic gradient $I_{v}$ can thus be decomposed into two parts: $I_{v}=I_{\text {stat }}+I_{f}$, with $I_{\text {stat }}$ the hydrostatic contribution and $I_{f}$ the wall shear-stress contribution. The hydrostatic contribution reads:

$$
I_{\text {stat }}=(s-1) \epsilon_{s}
$$

The in-situ concentration $\epsilon_{s}$ is a priori unknown. Following the seminal work of Newitt et al. 7], the average velocity difference between the solids and the surrounding water, or slip velocity $V_{\text {slip }}$ reads:

$$
V_{\text {slip }}=\frac{1-C}{1-\epsilon_{s}} V_{m i x}-\frac{C}{\epsilon_{s}} V_{m i x}
$$


This slip velocity would be the terminal velocity $V_{0}$ for a single solid of diameter $d_{p}$ and of drag coefficient $c_{d}=0.44$ falling in an infinite medium of fluid at rest:

$$
V_{0}=\sqrt{\frac{4 d_{p} g(s-1)}{3 c_{d}}}
$$

However, it must be corrected in the case of a concentrated mixture flowing in a pipe. Owing to the range of parameters that we are interested in, we have chosen to use the Richardson \& Zaki correlation [8] for the hindered average slip velocity:

$$
V_{\text {slip }}=\left(1-\epsilon_{s}\right)^{2.4} V_{0}
$$

The in-situ concentration is obtained by solving the non-linear system of Eqs. 1 and 3 in an iterative way, with a trust-region dogleg algorithm.

The model for the wall shear-stress contribution $I_{f}$ is based on some asumptions. As noticed by Engelmann [5] or Hong et al. 10], large particles tend to migrate away from the wall due to hydrodynamic lift 2]. Assuming that the near-wall velocity profile is only slightly affected by the presence of particles in the core region, the wall shearstress is modeled by water flowing at the water velocity:

$$
I_{f}=\lambda \frac{\left(V_{\operatorname{mix}} \frac{1-C}{1-\epsilon_{s}}\right)^{2}}{2 g D}
$$

This model for vertical ascending flow has been validated on various experimental data available in the Literature [9-11]. The comparison between the model and the data is plotted in Fig. 2. The agreement is very good. When dealing with a mixture of liquid and solids, the pressure drops are significantly higher than for pure fluid for the whole range of mixture velocity that corresponds here to $V_{0} \leq V_{\text {mix }} \leq 8 V_{0}$ : it is for instance twice as large at $C=5 \%$ and $V_{m i x}=4 V_{0}$ for the beads of diameter $d_{p}=0.2 D$ and $s=2.15$ (data of Yoon et al. 11], o in Fig. 2). A remarkable feature of the curves is the presence of a local minimum: the hydraulic gradient does not vary monotonically with the velocity. This is due to the fact that the hydrostatic gradient is the dominant term and that it is a decreasing function of the mixture velocity. In the case of ascending flows, the in situ concentration $\epsilon_{s}$ is indeed much larger than the delivered concentration $C$ for mixture velocities of the order of $V_{0}$ and decreases slowly with increasing $V_{m i x}$. For instance, for the data plotted with $\circ$ in Fig. 2. $V_{0}=0.83$ m.s ${ }^{-1}$ and the values of the computed in situ concentration are: $\epsilon_{s}=13 \%$ for $V_{\text {mix }}=V_{0}$, $\epsilon_{s}=11 \%$ for $V_{m i x}=1.2 V_{0}=1 \mathrm{~m} . \mathrm{s}^{-1}, \epsilon_{s}=10 \%=2 C$ for $V_{\text {mix }}=1.4 V_{0}$ and $\epsilon_{s}=6 \%$ for $V_{m i x}=4 V_{0}$. The contribution of the hydrostatic gradient to the total hydraulic gradient is respectively $96 \%, 93 \%, 90 \%$ and $43 \%$. The velocity which corresponds to the minimum of the pressure drop curve $\left(V_{m i x} \simeq 1.8 V_{0}\right.$ for $\circ$ in Fig. 2) is of great practical importance and the line should not be operated below this velocity.
The model is thus used with the parameters of the present experiments to compare the order of magnitude of hydraulic gradients and critical velocities between horizontal and vertical flows (see for instance Figs. 3 and $4 \mathrm{~b}$ ).

\subsection{Description of the typical regimes in horizontal pipes}

The Figure 3 presents a typical evolution of the hydraulic gradients $I_{h}$ and $I_{s}$ in the present experimental setup. The solids are Glass beads of diameter $5 \mathrm{~mm}$ and the delivered concentration is $C=5 \%$.

The thick black line in Fig. 3 stands for the measured hydraulic gradient in the horizontal part in the case with water flowing alone. The typical Reynolds number is $2 \times$ $10^{5}$ and the flow is fully turbulent. The curve is a fit of the form $I_{f}=\lambda \frac{V^{2}}{2 g D}$ that gives a value for the friction coefficients $\lambda_{h}=0.156$ and $\lambda_{s}=0.160$. Corresponding rugosities can be deduced with the Colebrook formula. The estimated rugosity is $20 \mu \mathrm{m}$ for the horizontal pipe.

The shape of the pressure drop curve with particles (red $\bullet: I_{h}$ and blue $\mathbf{\square}: I_{s}$ in Fig. 3) is very similar to the ascending vertical case with a large increase of the pressure drop for the whole range of tested mixture velocity (here, $V_{m i x} \leq 10.5 V_{0}$ ). The presence of a local minimum is also observed: the hydraulic gradient does not vary monotonically with the velocity. The mixture velocity for which the minimum hydraulic gradient is observed will be termed the critical velocity $V_{\text {crit }}$, following the definition of Doron et al. [12]. In the present case and in the horizontal pipe, the critical velocity is $V_{c r i t, h} \simeq 1.8 \mathrm{~m} . \mathrm{s}^{-1}$ (i.e. $V_{\text {crit }, h} \simeq 3.8 V_{0}$ ). All these observations are consistent with all the previous studies that dealt with similar large particles [4, 12, 14, 15].

For $V_{\text {mix }}<V_{\text {crit }}$ in the horizontal pipes, flow regimes with a stationary bed above which a compact layer of beads is flowing are observed (see bottom left picture in Fig. 3 that corresponds to $V_{m i x}=1.2 \mathrm{~m} . \mathrm{s}^{-1}$, i.e. $V_{m i x}=$ $\left.2.5 V_{0}\right)$. The more the mixture velocity decreases, the more the solids tend to settle down. These regimes are thus such that $\epsilon_{s}>C\left(\epsilon_{s} \simeq 32 \%\right.$ for bottom left picture in Fig. 3) and are characterized by a large pressure drop that is caused by a decrease in the discharge section. In the ascending part of the flexible pipe, a layer of solids located at the bottom of the pipe and that is flowing backwards is even observed for these low velocities, as already observed by Yamaguchi et al. 17] (see top left picture in Fig. 3 that is taken at $\left.V_{m i x}=0.9 \mathrm{~m} . \mathrm{s}^{-1}\right)$. The flow in this regime is very unstable and the transit time needed to reach a stationary state is very long, of the order of twenty minutes - the typical time for a solid to flow through the whole pipe being $30 \mathrm{~s}$.

Slightly above the critical velocity - for $V_{m i x} \gtrsim V_{c r i t}$ a moving bed on the bottom of the pipe is observed both in the horizontal pipe and in the S-shaped part (see bottom central picture and top right picture in Fig. 3 that are both taken at $V_{m i x}=2.1 \mathrm{~m} . \mathrm{s}^{-1}$, i.e. $\left.V_{m i x}=4.1 V_{0}\right)$. Very few beads are also transported by saltation. The mean 

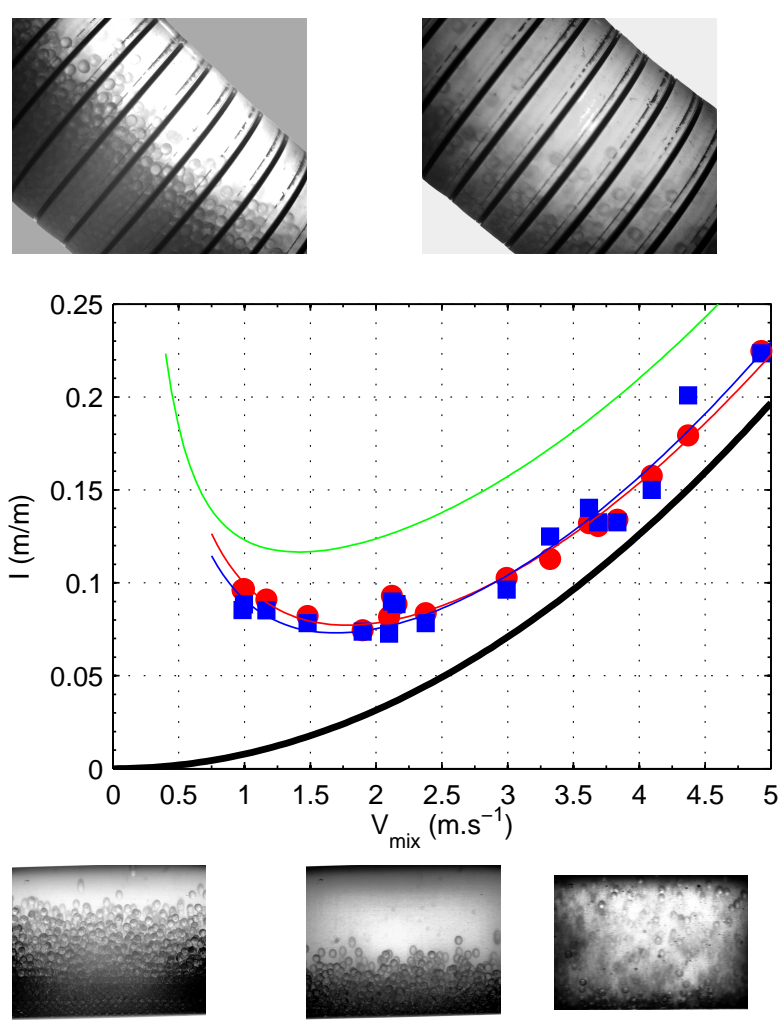

Figure 3: Illustration of the different regimes. Hydraulic gradient vs. $V_{m i x}$, Glass beads of $5 \mathrm{~mm}, C=5 \%$. Solid black line: water flowing alone $I_{f}$, red $\bullet: I_{h}$, blue $\mathbf{\square}: I_{s}$. The blue and red solid lines are fits of the type $a / V_{m i x}+b V_{m i x}^{2}$ (see $\S 3.3$ ). The green solid line stands for $I_{v}$ predicted with the model developed for vertical pipelines (see $\S 3.1$.

height of this moving bed is roughly $25 \mathrm{~mm}$ in the present case (Glass beads, $d_{p}=5 \mathrm{~mm}$ and $C=5 \%$ ), that corresponds to approximately five layers of beads. There is a velocity gradient between the upper layer of the moving bed and the lower layer that moves more slowly, as usually observed for hydraulic transport of sediment in twodimensional open channels 19]. Please note that there may be substantial differences between the present experiments and usual bed-load models for the transport of sediments [19], because of three-dimensional and geometrical effects due to the cylindrical section of the pipe and of the large particle diameter to pipe diameter ratio. The average velocity of this bed is small compared to the mixture velocity and $\epsilon_{s}>C$ here, $\epsilon_{s} \simeq 10 \%$.

Increasing further the mixture velocity, more and more solid beads get suspended and transported by the flow, there is no more bed, the flow is fully dispersed. The pressure drop curves behave as the clear-water pressure drop curve and follow the same trend at high velocities. In that case, $\epsilon_{s} \gtrsim C$ and the regime is called "pseudohomogeneous" 12] or "heterogeneous" [4] (see bottom right picture in Fig. 3 that is taken at $V_{m i x}=4.9 \mathrm{~m} . \mathrm{s}^{-1}$, i.e. $\left.V_{\text {mix }}=10.3 V_{0}\right)$.

The green line is the results of the model for pressure drop in ascending vertical flow, presented in $\S$ 3.1. The main contribution to the increase of the total hydraulic gradient in the vertical model comes from the hydrostatic pressure of the mixture. On the contrary, in the case of the horizontal pipe, the increase in the total hydraulic gradient originates in the bed formation that causes more friction. Some partial conclusions concerning the comparison of a vertical ascending pipe and an horizontal pipe in view of an application with complicated shapes can be drawn. Firstly, the hydraulic gradient in the horizontal part is lower than the one that would be observed in an ascending vertical pipe at least for this specific mass and solid size. Secondly, the critical velocity in the horizontal pipe is greater than that of a vertical pipe. The effects of size, concentration and specific mass that may be very different for a vertical and an horizontal pipe will be further explored in $\S$,

\subsection{Correlations and models}

This paragraph is a brief overview of some of the correlations and models that are commonly used.

Empirical correlations. The first quantity of interest to be presented is the critical velocity $V_{\text {crit }}$. One correlation has been proposed by Durand \& Condolios (1952) [14]:

$$
V_{\text {crit }}=F_{l}\{2 D g(s-1)\}^{1 / 2}
$$

with $F_{l}$ a constant of order unity, that depends on the delivered concentration and the particle size.

Concerning the prediction of the hydraulic gradient, a first empirical correlation is the one proposed by Durand \& coworkers [15]. They used sand particles of one specific mass with diameter up to $25.4 \mathrm{~mm}$ in pipes ranging from $38 \mathrm{~mm}$ to $558 \mathrm{~mm}$ in diameter (the maximum relative diameter in the pipe of $104 \mathrm{~mm}$ was $4.5 \%$ ). The dimensionless excess of pressure drop caused by the presence of the particles $\Phi$ is expressed using the two "Froude" numbers $F_{D}$ and $F_{d_{p}}$. These two parameters are grouped to form $\Psi=F_{D}^{2} F_{d_{p}}^{-1}$. They found a general correlation that best represents all their data:

$$
\Phi=180 C(\Psi)^{-1.5}
$$

It is recommended to use this correlation in the vicinity of the critical velocity (from mixture velocities slightly below to three or four times greater). This correlation was then modified to take into account the specific mass of the particles, as reported by Newitt et al. [4]. In the case of particles of a few millimeters flowing in water, the particle Reynolds number is sufficiently high to assume that the settling velocity is given by Eq. 2 with a constant drag coefficient $c_{d}$. This form of the correlation reads:

$$
\Phi=121 C\left(\frac{V_{m i x}^{2}}{g D(s-1)} \sqrt{\frac{3}{4} c_{d}}\right)^{-1.5}
$$

The functional form of $I_{h}$ that is predicted by this model is thus: $I_{h}=I_{f}\left(1+A V_{m i x}^{-3}\right)=b V_{m i x}^{2}+a V_{m i x}^{-1}$, as displayed in Figs. 3 and 6 . 
This correlation have been widely discussed [1, 2, 4, 12]. The main criticisms are that it does not take into account the size of the particles and that a single correlation may not be applicable to all flow regimes. The value of the constant that is reported in the Literature is moreover different according to various authors, the term $F_{d_{p}}^{-1}$ being sometimes abruptly replaced by $\sqrt{c_{d}}$.

Semi-empirical correlations. Newitt et al. [4] have lead experiments in a $25.4 \mathrm{~mm}$ in diameter horizontal pipe, with various particles covering four specific masses $(s=1.18$, $s=1.4, s=2.6$ and $s=4.1$ ) and various sizes, the largest being of equivalent diameter $2.6 \mathrm{~mm}$ (10\% of the pipe diameter). Theoretical considerations are used to derive expressions of the hydraulic gradient for different flow regimes (homogeneous, heterogeneous and flow with moving beds), and of the transition velocities between those regimes. For heterogeneous flows, i.e. for $17 V_{0} \leq V_{m i x} \leq$ $\sqrt[3]{1800 g D V_{0}}$, the excess of friction is considered proportionnal to the energy dissipated by the particles as they fall under the action of gravity. This hypothesis leads to:

$$
\frac{\Phi}{\epsilon_{s}} \propto(s-1) V_{0} V_{m i x}^{-3}
$$

For flows with a moving bed, i.e. for $\sqrt{2 g D(s-1)} \lesssim$ $V_{\text {mix }} \leq 17 V_{0}$, the effect of the solids is proportional to the solid-solid friction between the solids and the bottom of the pipe:

$$
\frac{\Phi}{\epsilon_{s}} \propto(s-1) V_{m i x}^{-2}
$$

The main asumption in Ref. [4] is the use of $C$ for $\epsilon_{s}$, that is equivalent to assume that the particles move at the same speed as the water or at a constant fraction of it. The suspension of particles through turbulence or Bagnold forces is moreover not taken into account. This is obviously not the case in the present experiment as can be seen with the pictures in Fig. 3 and the values of $\epsilon_{s} / C$ that are reported in $\S 3.2$.

Analytical model based on mass and momentum balance. Doron et al. 12 have established such a model. It is based on the decomposition of the cross-section of the pipe into two layers. It is thus a one dimensional model. The bottom of the pipe is assumed to be filled with a stationary or moving bed of packed particles. The height of this bed is $y_{b}$ and the volumetric concentration in this layer is $C_{b}=0.52$. All the particles are assumed to move at the same velocity in the bed. An heterogeneous mixture of solids and fluid is flowing in the upper part of the pipe. The mixture is treated as an homogeneous fluid with averaged physical properties and no slip between the phases is considered. The mass and momentum balance are then written in each layer. The shear stresses at the walls and at the interface between the two layers are modeled with frictions coefficients, and with a static friction force for the lower layer. In addition, the dispersion process of the solid particles in the upper layer is modeled by a turbulent diffusion process balanced by the gravitational settling of particles, leading to an advection-diffusion equation. The size of the particles is taken into account, firstly to define the roughness of the interface between the two layers, and secondly in the definition of the turbulent diffusion coefficient and the advection velocity that is the hindered terminal velocity (the Richardson \& Zaki correlation [8], Eq. 3] is used). This model leads to a non-linear system of 5 equations with 5 unknowns: the bed height $\left(y_{b}\right)$, the velocity of the upper layer $\left(U_{h}\right)$, the velocity of the lower layer $\left(U_{b}\right)$, the concentration in the upper layer $\left(C_{h}\right)$ and the pressure gradient $(\nabla P)$. The parameters of the model are: the solid friction coefficient between the pipe wall and the particles $(\eta)$, an angle of internal friction that models the normal stress transmitted by the shear stress at the interface between the fluid and the bed $(\varphi)$, the packing concentration $\left(C_{b}\right)$ and the correlations for fluid friction coefficients. This model have been implemented in Matlab, using an iterative procedure with a trust-region dogleg algorithm to solve the non-linear system. This implementation has been validated on the data of Ref. [12] in Fig. 4f.

\section{Results and discussion}

\subsection{Effects of the physical characteristics of the beads}

This paragraph is devoted to the comparison of the pressure drop curves with various concentrations, specific masses and sizes for identical spherical beads. The reference case is the Alumina beads of diameter $6 \mathrm{~mm}$, of specific mass $s=3.65$, and at a delivered concentration $C=5 \%$. In this case, the order of magnitude of the minimal pressure drop at critical velocity $V_{\text {crit }, h} \simeq 2.4 \mathrm{~m} . \mathrm{s}^{-1}$ is $I_{\text {crit }, h} \simeq 0.11 \mathrm{~m} / \mathrm{m}$.

The effects of the concentration are presented in Fig. 4ab. Only results for the horizontal part are plotted. On the one hand, increasing the delivered concentration leads to an increase of the pressure drop, as expected. The relative increase of the pressure drop at the critical point with respect to $C=5 \%$ is roughly $40 \%$ for $C=10 \%$ and $70 \%$ for $C=15 \%$. Very few points are available for the concentration $C=20 \%$ owing to the large power required; these points are nevertheless on both sides of the critical point. The relative increase in pressure drop at the critical point for $C=20 \%$ is around $100 \%$. On the other hand, changing the concentration seems to increase only very slightly the critical velocity (see $\S 4.3$ for a discussion of this point).

The comparison of two sizes of beads of same specific mass at the same concentration is presented in Fig. 45$\mathrm{d}$ : the experimental measurements for Alumina beads of diameter $d_{p}=6 \mathrm{~mm}$ are plotted with $\circ$ and the results for Alumina beads of diameter $d_{p}=15 \mathrm{~mm}$ are plotted with $\square$. The pressure drop that would be observed in an ascending vertical pipe according to the prediction of the model (see $\S$ 3.1) is plotted with, respectively a solid red line for $d_{p}=6 \mathrm{~mm}$ and a dashed red line for $d_{p}=$ 

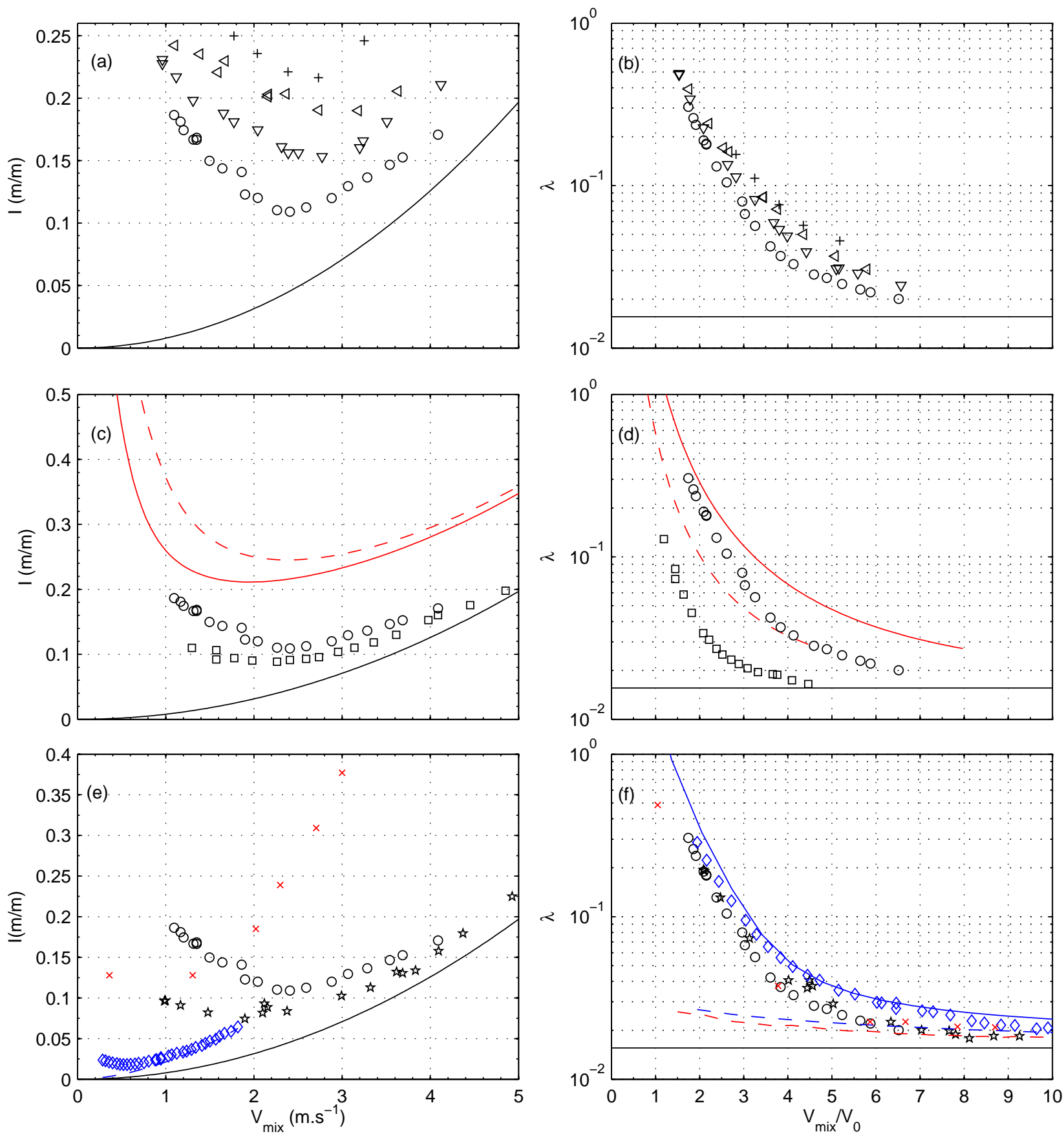

Figure 4: (a,c,e): Hydraulic gradient $I_{h}$ vs. mixture velocity $V_{\text {mix }}$. (b,d,f): dimensionless plot of $\lambda_{h}$ vs. $V_{m i x} / V_{0}$.

(a-b): Experimental data for Alumina beads of $d_{p}=6 \mathrm{~mm}$ at various concentrations: $C=5 \%(\circ), C=10 \%(\nabla), C=15 \%(\triangleleft)$ and $C=20 \%$ $(+)$. Solid black line: water flowing alone.

(c-d): Experimental data in the horizontal pipe (symbols) and predictions of the model presented in $\S 3.1$ for a similar vertical pipe (red lines), for Alumina beads at $C=5 \%$ and two sizes: $d_{p}=6 \mathrm{~mm}$ ( $\circ$ and solid red line) and $d_{p}=15 \mathrm{~mm}$ ( $\square$ and dashed red line).

(e-f): Experimental data for $d_{p}=5 \& 6 \mathrm{~mm}$ and $C=5 \%$ for two specific masses: Alumina (o) and Glass ( $\star$ ). Blue $\diamond$ : data extracted from Fig. 3 of Ref. [12] (particles of diameter $d_{p}=3 \mathrm{~mm}, s=1.24$ flowing in an horizontal pipe of diameter $D=50 \mathrm{~mm}$ at $C=4.2 \%$ ), solid blue line: validation of the two-layer model, dashed blue line: single phase pressure drop. Red $\times$ : data extracted from Fig. 12 of Ref. [4] (gravels of mean diameter $d_{p}=2.6 \mathrm{~mm}, s=2.55$ flowing in an horizontal pipe of diameter $D=25.4 \mathrm{~mm}$ at $C=5 \%$, red dashed line: single phase pressure drop. 
$15 \mathrm{~mm}$. The main effects of the size are first that the pressure drop is decreased for the large particles in the horizontal pipe, and secondly that the critical velocity does not seem to be affected by the particle size. This feature is quite surprising to the best of our knowledge has not been reported in previous works that mainly dealt with particles below $4 \mathrm{~mm}$ (see $\S 3.3$ ). It is moreover robust as it is confirmed on the Glass beads (see $\star$ and $\diamond$ in Fig. 86). The decrease of pressure drop with larger particles and the constancy of the critical velocity are a distinguishing feature between horizontal and vertical flows: owing to the dependence of the slip velocity on the square root of the particle diameter, the hydraulic gradient and the critical velocity are greater for larger solids in vertical flows as a direct result of the model presented in $\S 3.1$.

Finally, the experimental measurements of the pressure drop for two beads of similar size $\left(d_{p} \simeq 5 \mathrm{~mm}\right)$ and two different specific masses, respectively $s=3.65$ (०) and $s=$ $2.5(\star)$ are plotted in Fig. 44-f. Increasing the specific mass leads to both an increase of the pressure drop and of the critical velocity. Experimental data on particles of similar size that are available in the Literature [4, 12] are also plotted in this figure. Please note that they have been collected in pipes of smaller diameters. The shape of the present curves is very similar to previously reported works, particularly when presented in a dimensionless form $(\lambda v s$. $V_{m i x} / V_{0}$, in Fig. $\left.4 \mathrm{f}\right)$. Please nonetheless notice that the single-phase flow pressure drop coefficients are different (black solid line, blue and red dashed lines in Fig. 4f $)$.

\subsection{Mixes of beads and rough solids}

This paragraph deals with mixtures of spheres and rough stones of arbitrary shapes in order to check to what extent the results obtained for mono-disperse spheres may represent an actual application.

The figure 5 presents the horizontal hydraulic gradient $I_{h}$ vs. $V_{m i x}$ for different mixtures of Alumina beads of diameter $6 \mathrm{~mm}$ and $15 \mathrm{~mm}$. Contrary to what one might think a priori, the pressure drop of the mixtures is not a simple linear combination of the pressure drop of each bead size: for a $50 \%$ of $6 \mathrm{~mm}$ mixture (mixture 1) the pressure drop curve coincides with that of the $15 \mathrm{~mm}$ beads. The pressure drop is thus low. This effect is even still present for a proportion of $75 \%$ of $6 \mathrm{~mm}$ beads in the mixture (mixture 2) but only at low mixture velocities corresponding to $V_{m i x} \lesssim V_{c r i t}$, i.e. to regimes with a stationary bed. For higher velocities the pressure drop lies between the other two and is closer to the pressure drop of the $6 \mathrm{~mm}$ beads.

The figure 6 presents the comparison of $I_{h}\left(V_{m i x}\right)$ for three mixtures of beads of same size but different specific mass: Glass beads of $5 \mathrm{~mm}(\star)$, Alumina beads of $6 \mathrm{~mm}$ (০) and mixture $3(50 \%$ Glass / 50\% Alumina, $\triangleleft)$. The pressure drop curve for the mixture lies between the two single-type cases and seems to be well modelized by the mean of the two curves: the solid red line in Fig. [6 is a fit
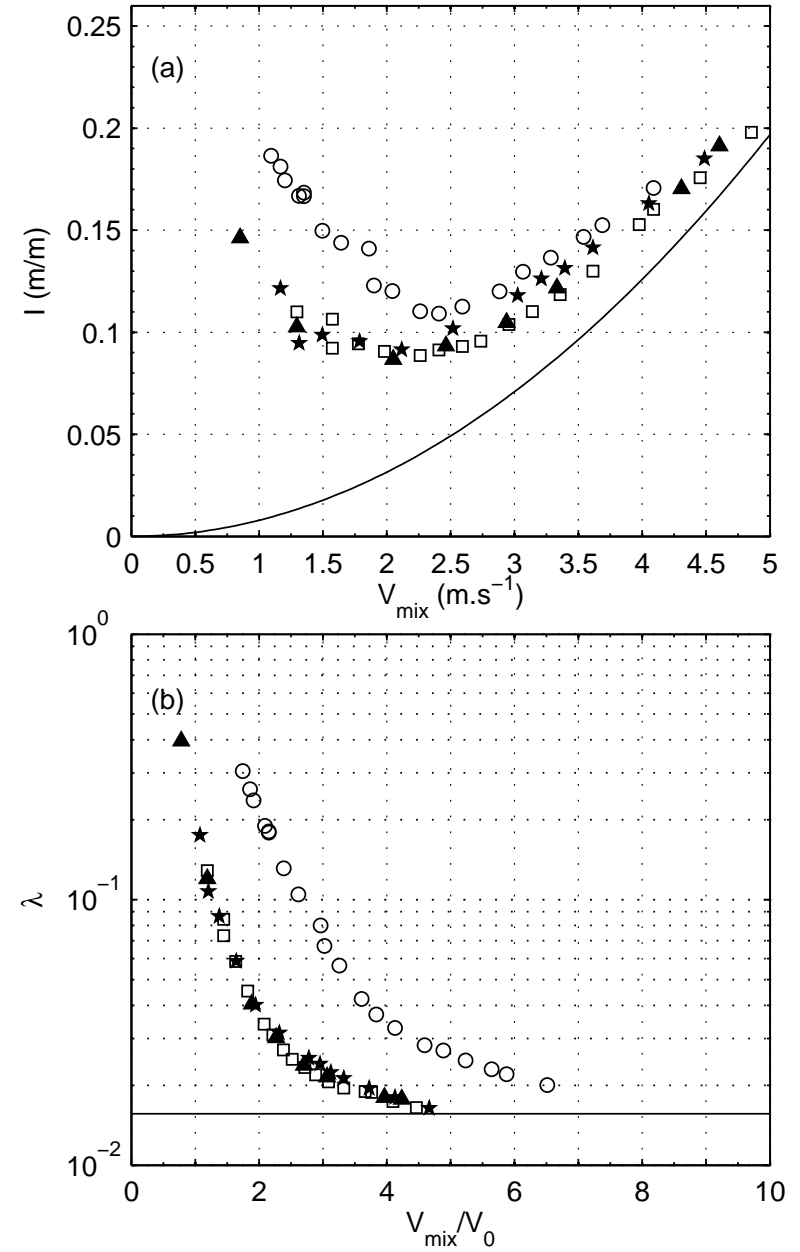

Figure 5: (a): Hydraulic gradient $I_{h}$ vs. mixture velocity $V_{\operatorname{mix}}$ and (b): dimensionless plot of $\lambda_{h}$ vs. $V_{m i x} / V_{0}$, at $C=5 \%$ vs. for ○: Alumina $6 \mathrm{~mm}, \square$ : Alumina $15 \mathrm{~mm}, \triangle$ : mixture 1 and $\star$ : mixture 2 .

for the Alumina, the solid blue line a fit for Glass and the black line is the mean of these two curves.

The effects that have been observed for mixtures, particularly in the horizontal part, may be ascribed to segregation phenomena. This is illustrated in Fig. 7 for mixture 2 of Alumina of two sizes (see Tab. 1). A tendency of having two layers of beads, with the small beads being transported at the bottom of the pipe while the large beads are transported on top of this bed, is indeed observed. This phenomenon is reminiscent of the so-called "Brazil nut effect". Likewise, for the mixture of beads of different specific masses, the heaviest tend to settle at the bottom of the pipe.

All the previous results concern experiments with spherical beads of unique size and specific mass or mixtures of at most two different types of spherical beads. The pressure drop curves for stones are plotted in Fig. 8. Their physical characteristics are given and illustrated in Tab. 1. The specific mass of a sample of fifty solids have been measured with a densimeter. It is constant within $2700 \pm 10 \mathrm{~kg} \cdot \mathrm{m}^{-3}$. Their specific mass is thus very close to that of Glass. 

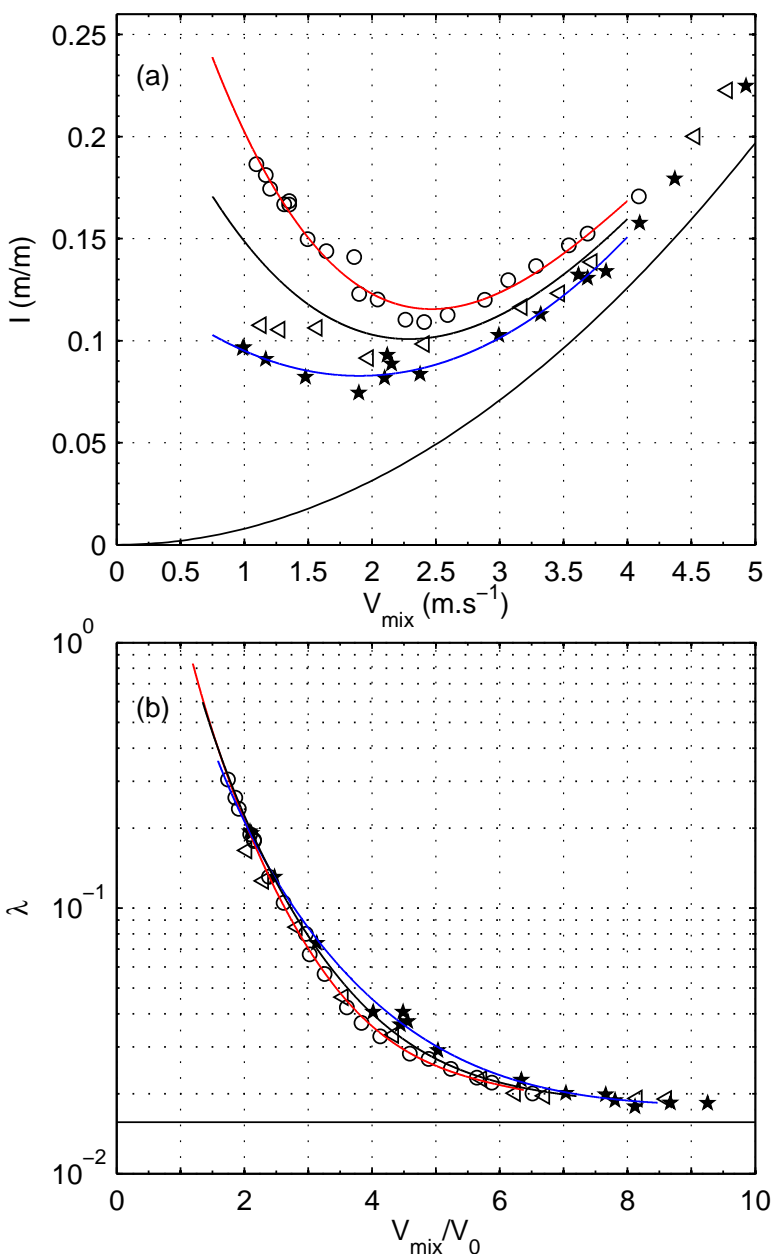

Figure 6: (a): Hydraulic gradient $I_{h}$ vs. mixture velocity $V_{m i x}$ and (b): dimensionless plot of $\lambda_{h}$ vs. $V_{\operatorname{mix}} / V_{0}$, at $C=5 \%$ for $\circ$ : Alumina $6 \mathrm{~mm}$ with a fit corresponding to the red line, $\star$ : Glass $5 \mathrm{~mm}$ with a fit corresponding to the blue line and $\triangleleft$ : mixture 3 . The black solid line is the mean of the two fits.

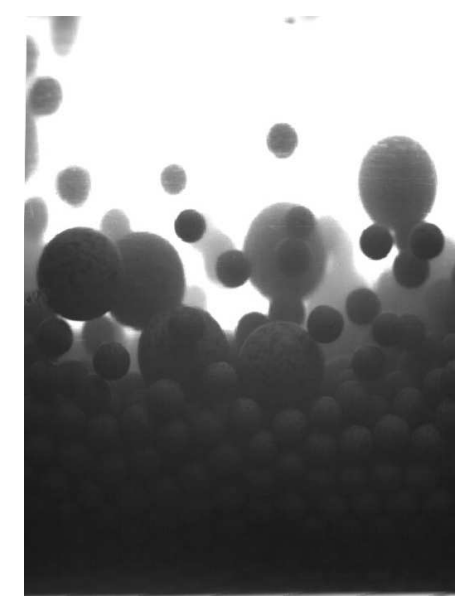

Figure 7: Illustration of segregation for mixture 2. In that case, $C=5 \%$ and $V_{m i x} \simeq 1 \mathrm{~m} . \mathrm{s}^{-1}$.
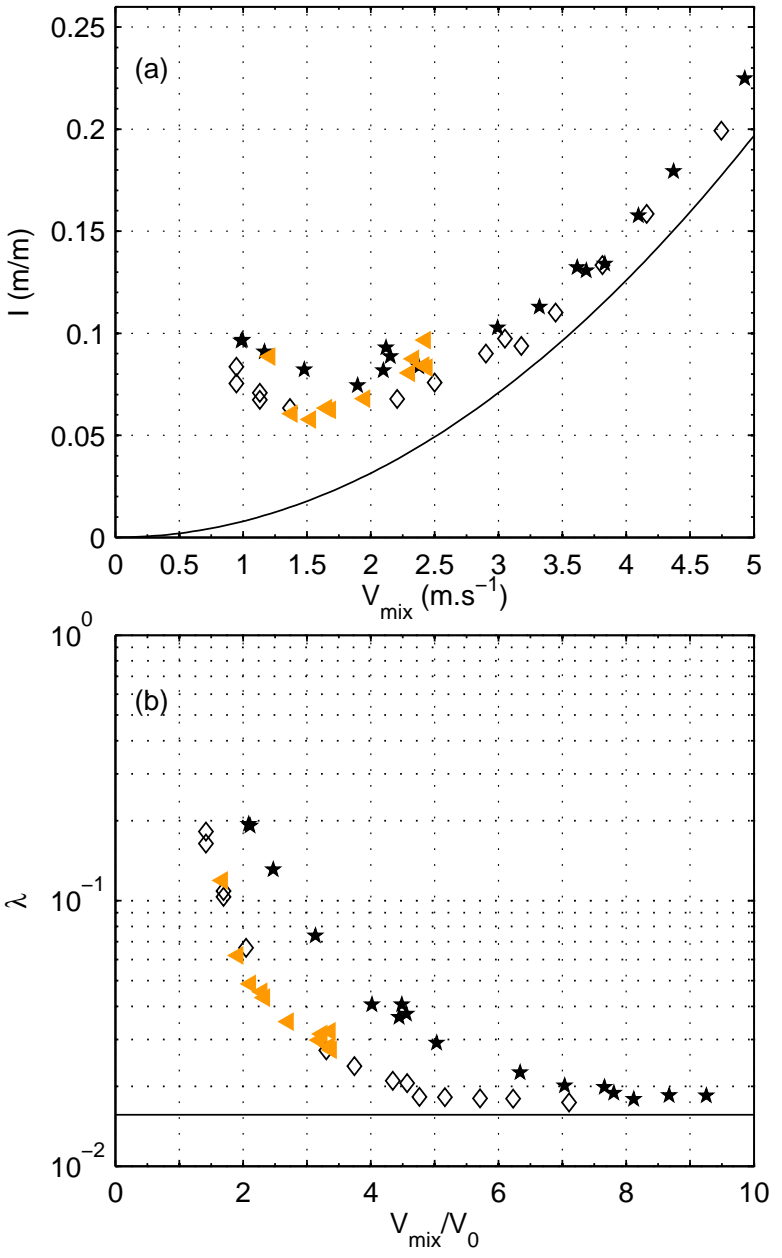

Figure 8: (a): Hydraulic gradient $I_{h}$ vs. mixture velocity $V_{m i x}$ and (b): dimensionless plot of $\lambda_{h}$ vs. $V_{m i x} / V_{0}$, at $C=5 \%$ for $\star$ : Glass $5 \mathrm{~mm}, \diamond$ : Glass $10 \mathrm{~mm}$ and orange $\triangleleft$ : stones.

Their size distribution is between 8 and $18 \mathrm{~mm}$, with $50 \%$ of the solids having a size lower than $10 \mathrm{~mm}$. The pressure drop $I_{h}$ for these solids is very close to the case of $10 \mathrm{~mm}$ Glass beads. The irregular shape of the solids that leads to different drag coefficients thus does not seem to play an important role with respect to the hydraulic gradient, as already reported by Yoon et al. [11]. It may be a second order effect with respect to the size and specific mass effects.

\subsection{Analysis and discussion}

This section is devoted to the application of various models to the present data and to their discussion.

Semi-empirical correlations. The first quantity that could be checked is the critical velocity $V_{\text {crit }}$ that is the mixture velocity for which the minimum hydraulic gradient is observed. The present results suggest that the critical velocity does not strongly depend on the particle size but depends on the specific mass (Fig. 4k-e). The terminal settling velocity furthermore does not seem to be the velocity 
scale that drives the pressure drop curve as can be seen in Fig. 4d. The correlations that use the "Froude" number $F_{D}$ (Eqs. 4 and 5) may be applicable.

The results concerning the prediction of the critical velocity are reported in Tab. 2, The predicted values are in excellent agreement with the experimental values for Glass and Alumina beads of various sizes and at various concentrations (see Figs. 4 for Alumina and 8 for Glass).

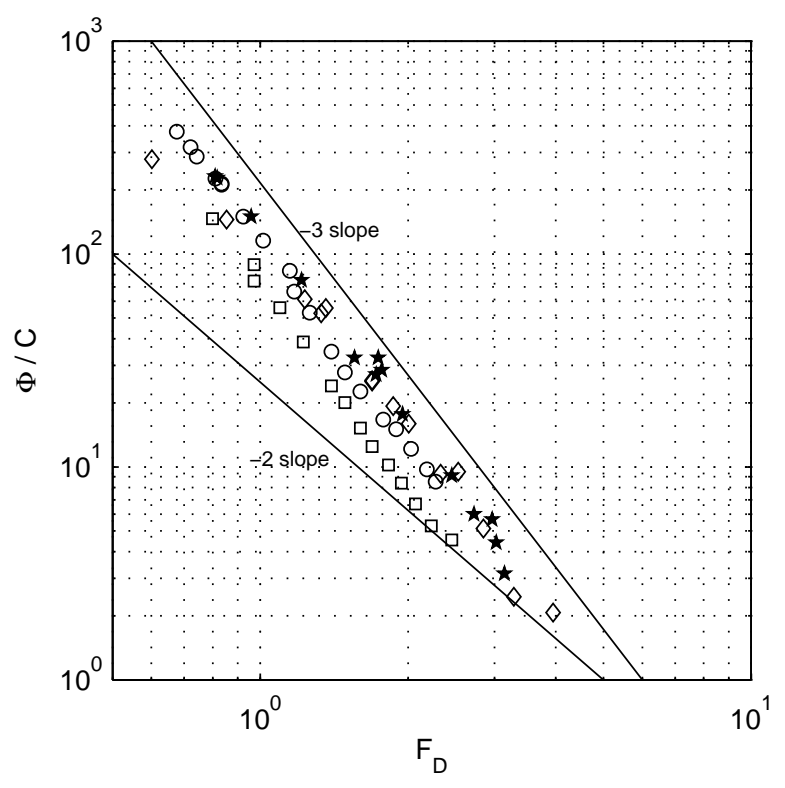

Figure 9: Dimensionless excess of head loss divided by the delivered concentration $\Phi / C$ vs. $F r$ for $\star$ : Glass beads of $5 \mathrm{~mm}(C=5 \%)$, $\diamond$ : Glass beads of $5 \mathrm{~mm}(C=10 \%)$, ०: Alumina beads of $6 \mathrm{~mm}$ $(C=5 \%)$ and $\square$ : Alumina beads of $15 \mathrm{~mm}(C=5 \%)$.

The value of the dimensionless excess of head loss $\Phi$, normalized by the delivered concentration $C$ is plotted as a function of the Froude number $F_{D}$ for various data sets in Fig. 9. All the data follow the same trend. The effect of the concentration is moreover well described by a linear dependence: the data points for Glass beads of $5 \mathrm{~mm}$ at a concentration $C=5 \%(\star)$ and at a concentration $C=10 \%(\diamond)$ collapse on a single curve. A -3 power law $\Phi=C K F_{D}^{-3}$ fits quite well the data in the range $0.7 \lesssim F_{D} \lesssim 3$. The effect of specific mass that is included in the definition of the Froude number seems to be well taken into account: the data points for Glass beads of $5 \mathrm{~mm}$ at a concentration $C=5 \%, C=10 \%(\star \& \diamond)$ and for Alumina beads of $6 \mathrm{~mm}$ at a concentration $C=5 \%$ (०) are very close, the values of the constant $K$ being respectively $130 \pm 4$ and $123 \pm 3$. All these considerations are consistent with the empirical correlation (Eq. 5) and the theoretical equation (Eq. 6) with $V_{0} \propto \sqrt{s-1}$ for large particles. On the contrary, the -2 power law and the dependency in $(s-1)$ of $\Phi$ that are predicted by Eq. 7 does not seem to be consistent with the present measurements. As already noticed, the size of the particles, that is not taken into account in the model, has a strong influence on the hydraulic gradient: the value of the constant for
Glass beads of $10 \mathrm{~mm}$ (not represented in Fig. 9) is indeed $K=87$ and the value for Alumina beads of $15 \mathrm{~mm}(\square)$ is $K=75$. This is predicted neither by Eq. 5 (no dependence in $\left.d_{p}\right)$ nore by Eq. 6 ( $\Phi$ increases as $\left.\sqrt{d_{p}}\right)$. The model that leads to Eqs. 6 and 7 relies on the hypothesis that $\epsilon_{s} \propto C$. This is not the case in the present experiements: for Glass beads of $d_{p}=5 \mathrm{~mm}$ at $C=5 \%$, the values of the in situ concentration estimated with imaging are indeed: $\epsilon_{s} \simeq 32 \%, 10 \%$ and $7 \%$ for $V_{\text {mix }} / V_{\text {crit }, h}=0.67$, 1.17 and 2.72. For the Alumina beads of $d_{p}=6 \mathrm{~mm}$ and $d_{p}=15 \mathrm{~mm}$, the measured concentrations are $\epsilon_{s} \simeq 11 \%$ and $9 \%$ for $V_{m i x} / V_{c r i t, h}=0.83$ and 1.66 .

Analytical model based on mass and momentum balance. The question that is adressed is to what extent the analytical model of Doron et al. [12] may apply to the present physical parameters, with solids of much higher specific mass and even higher relative diameter.

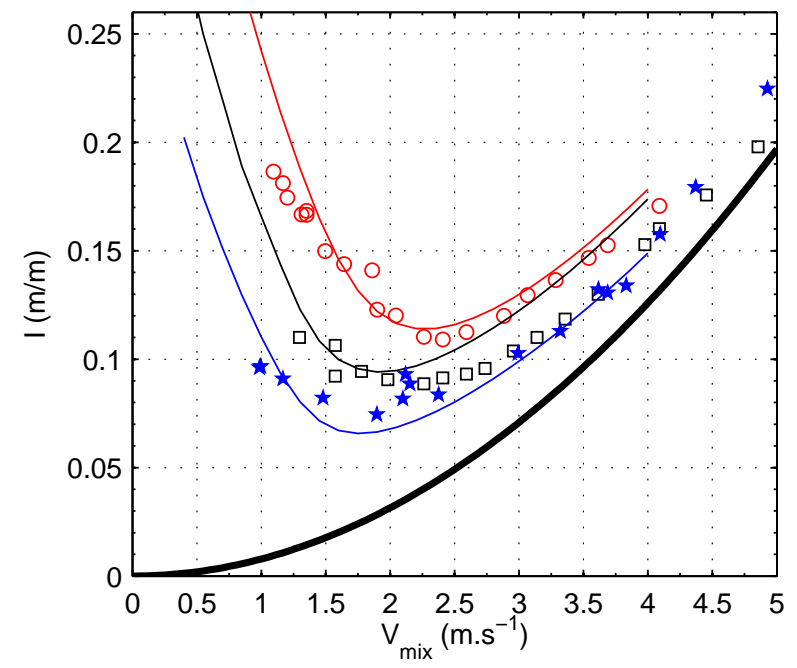

Figure 10: Results of the model (solid lines) compared to the present experimental data (symbols) at $C=5 \%$. Blue line and $\star$ : Glass $5 \mathrm{~mm}$. Red line and ०: Alumina $6 \mathrm{~mm}$. Black line and $\square$ : Alumina $15 \mathrm{~mm}$. Parameters for the model: $C_{b}=0.52, \eta=0.25, \tan \varphi=\infty$ (see text).

In the original work of Doron et al. [12], the values of the parameters are the following: $\eta=0.3, \tan \varphi=0.6$ and $C_{b}=0.52$. The packing concentration $C_{b}$ has first been experimentally measured by weighting a tube of same diameter and capacity two liters filled with dry beads and with beads and water. A dozen of measurements have been performed for each type of bead. The concentration is found to be $0.52 \pm 0.01$. The determination of the solid friction coefficient for an immersed granular bed is a very difficult problem 20], and the friction coefficient itself can strongly vary with the beads roughness 21]. We thus choose to vary $\eta$ and to present the results that better match the experimental data in the vicinity of the critical velocity. The angle $\varphi$ is set to $\varphi=\pi / 2$ which corresponds to neglecting the contribution of the normal stress transmitted into the bottom layer by the shear at the interface. 


\begin{tabular}{c|c|c}
\hline \hline & Glass $5 \mathrm{~mm}, C=5 \%$ & Alumina $6 \mathrm{~mm}, C=5 \%$ \\
\hline Experiment & 1.8 & 2.4 \\
\hline Eq. [4 with $F_{l}=1$ & 1.7 & 2.3 \\
\hline Eq. [4 with $F_{l}=1.05$ & 1.8 & 2.4 \\
\hline \hline
\end{tabular}

Table 2: Critical velocity $V_{\text {crit }}\left(\mathrm{m} . \mathrm{s}^{-1}\right)$ experimentally measured for Alumina and Glass beads, compared to the values predicted with the correlation 4

This asumption is validated a posteriori by evaluation of its relative contribution.

The results for the hydraulic gradient are presented in Fig. 10. The final value of the friction coefficient is $\eta=$ 0.25 . The predictions of the model for the two small beads of different specific mass and relative diameter $5 \%$ are in relatively good accordance with the experiments. For the largest beads, the trend of the reduction of the hydraulic gradient is reproduced but the model is not satisfactory: it matches the data point only very close to the critical velocity.
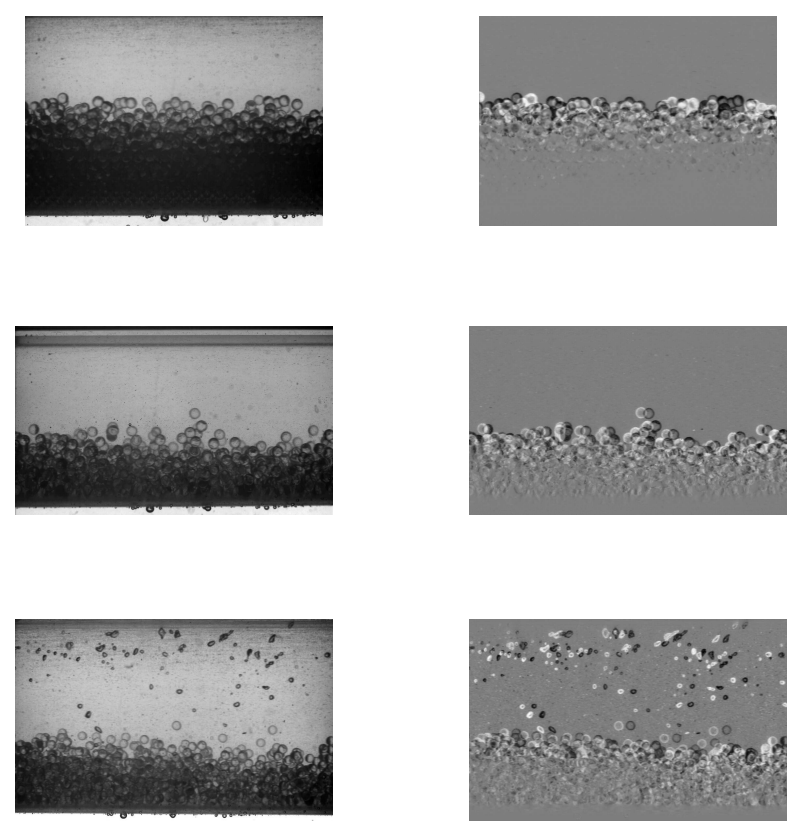

Figure 11: Pictures taken in the horizontal pipe of Glass beads of diameter $5 \mathrm{~mm}$ at $C=5 \%$. Left column: rough picture. Right column: difference between two successive images taken at the frame rate $f$. From top to bottom: $V=1.0 \mathrm{~m} . \mathrm{s}^{-1} \& f=50 \mathrm{~Hz}, V=$ $1.9 \mathrm{~m} . \mathrm{s}^{-1} \& f=500 \mathrm{~Hz}$ and $V=3.9 \mathrm{~m} \cdot \mathrm{s}^{-1} \& f=630 \mathrm{~Hz}$.

The model also predicts the height of the lower layer and the velocities of the two layers. Some pictures taken for the Glass beads at $C=5 \%$ are presented in Fig. 11 at different velocities. The right column corresponds to the difference between two successive images and allows to better identify the different flow regimes and the velocity gradient in the bed. At $V=1.0 \mathrm{~m} \cdot \mathrm{s}^{-1}$, one can clearly identify a static bottom layer and a packed layer of 3 to 4 beads that are transported with almost no vertical gradient. The height of the static bed is roughly $42 \mathrm{~mm}$ while the value predicted by the model is $40 \mathrm{~mm}$. At $V=1.9 \mathrm{~m} \cdot \mathrm{s}^{-1}$, the bottom layer is moving slowly with almost no vertical gradient of velocity. The height of the moving packed bed is roughly $25 \mathrm{~mm}$ while the value predicted by the model is $22 \mathrm{~mm}$. Above this moving bed, an unpacked layer of suspended beads is observed. And finally, at $V=3.9 \mathrm{~m} \cdot \mathrm{s}^{-1}$, one can still distinguish two layers of solids, with a bottom layer that is moving more rapidly. The height of this static bed is roughly $22 \mathrm{~mm}$ while the value predicted by the model is $15 \mathrm{~mm}$. The velocities that are evaluated with the movies give similar accordance.

In conclusion, this two-layer model, originally validated only on small specific mass particles, is promising and predicts well the global and few local features of the two-phase flow for larger specific masses. It seems however limited to not too large particles. One question is the validity of the advection-diffusion equation that is used to determine the concentration in the upper layer for particles that become larger than the turbulent eddies.

\section{Conclusion}

The hydraulic gradient as a function of mixture velocity has thus been measured for calibrated beads of two specific masses $\left(2500 \mathrm{~kg} \cdot \mathrm{m}^{-3}\right.$ and $\left.3650 \mathrm{~kg} \cdot \mathrm{m}^{-3}\right)$, with large particle to pipe diameter ratios of 5,10 and up to $15 \%$, as well as for mixtures of calibrated beads and for real stones of similar physical properties. The tests have been conducted in an horizontal pipe. The main results are the following:

- The increase of friction in an horizontal pipe is quite large at velocities of the order of the terminal velocity of a settling particle. This increase of the hydraulic gradient is however lower than that which would be observed because of the hydrostatic contribution in an ascending vertical pipe. On the other hand, the critical velocities that are a key parameter with respect to plugging for the design of a complex industrial application are greater.

- For a given specific mass and delivered concentration, the hydraulic gradient decreases with the increase of the particle size in an horizontal pipe, contrary to what is observed in vertical pipes.

- The empirical correlations that are available in the Literature give satisfactory results but the classical 
constants that are recommended clearly do not correspond to the present case of very large particles. Further studies with even larger beads are necessary to better model the variation of these constants with the particle size.

- The mixtures and real stones could be modeled with mono-dispersed beads of equivalent specific mass and size. However, at low velocities, strong segregation mechanisms are present and would make the modelisation harder.

- Analytical models based on more physical arguments are of great interest. They however show their limits for the largest beads.

This last point may be linked to the fact that for large beads the modelisation of the solids using a continuous medium even with the help of granular theory becomes very untrustworthy. The number of particles that can be put in the pipe cross-section is too small to be treated with statistical methods. For instance, numerical methods with Eulerian-Eulerian formulation such as the one presented in Ref. 22 is unappropriate to our case and even give unpresentable results. Full Lagrangian methods would also be very expensive owing to the nevertheless large number of particles. We are now developing two alternate methods: one is based on Lagrangian dynamics for the solids, the forces being prescribed with the help of a two-layer global model for the fluid. The idea is to detect the position of the compact bed and to compute the concentration in the upper layer, and then to apply some bed-load sediment transport equations [19] for the momentum balance in the bed. The second is to develop a Navier-Stokes code that uses volume penalization [23] that seem very promising to cope with the solids.

Further experiments are scheduled in the S-shape part, and in inclined pipes.

\section{Acknowledgement}

We would like to thank the Technip company for financial support, especially J. Denegre, P. Espinasse and T. Parenteau. F. R. thanks Colette Gauthier for fruitful discussion. We are particularly indebted to M. Joulin from the DynFluid laboratory of Arts et Metiers ParisTech, as well as to the students J.-R. De Céa and C. Valet for their assistance in the design and development of the experiment. The main part of the experiments have been carried out with the help of A. Lemaire.

[1] P.E. Baha Abulnaga. Slurry Systems Handbook. McGraw-Hill, 2002.

[2] K. C. Wilson, G. R. Addie, A. Sellgren, and R. Clift. Slurry Transport Using Centrifugal Pumps. Springer, 2006.

[3] V. Matousek. Predictive model for frictional pressure drop in settling-slurry pipe with stationary deposit. Powder Technology, 192:367-374, 2009.

[4] D. M. Newitt, J. F. Richardson, M. Abbott, and R. B. Turtle. Hydraulic conveying of solids in horizontal pipes. Trans. Instn. Chem. Engrs., 33:93-110, 1955.
[5] H. E. Engelmann. Vertical hydraulic lifting of large-size particles - a contribution to marine mining. In $10^{\text {th }}$ Off. Tech. Conf., pages 731-740, 1978.

[6] K. Pougatch and M. Salcudean. Numerical modeling of deep sea air-lift. Ocean Engineering, 35:1173, 2008.

[7] D. M. Newitt, J. F. Richardson, and B. J. Gliddon. Hydraulic conveying of solids in vertical pipes. Trans. Instn. Chem. Engrs., 39:93-100, 1961 .

[8] J. F. Richardson and W. N. Zaki. Sedimentation and fluidisation. Trans. Inst. Chem. Engrs., 32:35-53, 1957.

[9] J. X. Xia, J. R. Ni, and C. Mendoza. Hydraulic lifting of manganese nodules through a riser. J. Offshore Mechanics and Arctic Engineering, 126:72, 2004.

[10] S. Hong, J. Choi, and C. K. Yang. Experimental study on solid-water slurry flow in vertical pipe by using ptv method. In Proceedings of the Twelfth (2002) International Offshore and Polar Engineering Conference, pages 462-466, 2002.

[11] C. H. Yoon, J. S. Kang, Y. C. Park, Y. J. Kim, J. M. Park, and S. K. Kwon. Solid-liquid flow experiment with real and artificial manganese nodules in flexible hoses. In Proceedings of the Eighteenth (2008) International Offshore and Polar Engineering Conference, pages 68-72, 2008.

[12] P. Doron, D. Granica, and D. Barnea. Slurry flow in horizontal pipes - experimental and modeling. Int. J. Multiphase Flow, 13:535-547, 1987.

[13] P. Doron, M. Simkhis, and D. Barnea. Flow of solid-liquid mixtures in inclined pipes. Int. J. Multiphase Flow, 23:313323, 1997.

[14] R. Durand and E. Condolios. Experimental investigation of the transport of solids in pipes. In Deuxième Journée de lhydraulique, Societé Hydrotechnique de France, 1952.

[15] R. Durand. Basic relationship of the transportation of solids in pipes - experimental research. In Proc. Minnesota International Hydraulics Conference, pages 89-103, 1953.

[16] V. Matousek. Pressure drops and flow patterns in sand-mixture pipes. Experimental thermal and fluid science, 26:693-702, 2002.

[17] H. Yamaguchi, X.-D. Niu, S. Nagaoka, and F. de Vuyst. Solidliquid two-phase flow measurement using an electromagnetically induced signal measurement method. J. Fluids Eng., 133:041302, 2011.

[18] M. Van de Velden, J. Baeyens and K. Smolders. Solids mixing in the riser of a circulating fluidized bed. Chemical Engineering Science, 62:2139-2153, 2007.

[19] R. A. Bagnold. The flow of cohesionless grains in fluids. Philosophical Transactions of the Royal Society of London. Series A, Mathematical and Physical Sciences, 249:235-297, 1956.

[20] T. Divoux and J.-C. Géminard. Friction and dilatancy in immersed granular matter. Physical review letters, 99:258301, 2007.

[21] N. A. Pohlman, B. L. Severson, J. M. Ottino, and R. M. Lueptow. Surface roughness effects in granular matter: Influence on angle of repose and the absence of segregation. Physical Review E, 73:031304, 2006.

[22] J. Ling, P. V. Skudarnov, C. X. Lin, and M. A. Ebadian. Numerical investigations of liquid-solid slurry flows in a fully developed turbulent flow region. Int. J. Heat and Fluid Flow, 24:389-398, 2003.

[23] D. Kolomenskiy and K. Schneider. A fourier spectral method for the navier-stokes equations with volume penalization for moving solid obstacles. Journal of Computational Physics, 228:56875709, 2009. 